\title{
Band Alignment in Van der Waals Phosphorous Heterostructures
}

\author{
Daniel Hashemi, Michael Snure, and Stefan C. Badescu \\ Air Force Research Laboratory \\ Wright-Patterson AFB, OH, United States \\ daniel.hashemi.ctr@us.af.mil; michael.snure.1@us.af.mil; catalin.badescu.1@us.af.mil
}

\section{Extended Abstract}

Two-dimensional (2D) materials including graphene and phosphorene are attracting extensive attention due to their outstanding properties and wide range of applications in electronic and optoelectronic devices. Few layer black phosphorous (BLK-P) has been fabricated through exfoliation from the bulk BLKP. Layered BLK-P exhibits a range of direct band gaps $(0.3$ to $1.5 \mathrm{eV})$ [1] making it appealing for applications in optoelectronics. From layered BLK-P's interesting electronic properties, a carrier mobility up to $1000 \mathrm{~cm}^{2} / \mathrm{V}$.s [2] has been reported and an on/off ratio up to $10^{4}$ was achieved for phosphorene transistors at room temperature. Van der Waals (vdW) heterostructures based on BLK-P have presented great opportunities for application in nanoelectronics. For instance, the Schottky barrier height and doping of BLK-P can be controlled by electrostatic gating in graphene/BLKP heterostructures [3] and BLK-P/MoS 2 p-n junctions show strong gatetunable current-rectifying I-V characteristics. [4] Single-element vdW heterostructures composed of BLK-P and violet phosphorous (V-P) can be observed during bulk synthesis [5] or through decomposition of BLK-P.

Accurate modeling tools can help predict and assess the band alignment in these novel systems and at their interfaces with encapsulating materials like Boron nitride $(\mathrm{BN})$, which protect the conduction channel via encapsulation with widebandgap quasi-2D materials. Understanding of band alignment in these heterostructures is of great importance for tuning their electronic properties in potential nanoelectronic device applications. Here, we investigate the bandgaps of several materials and their band alignments using accurate density functional theory (DFT) with the Heyd-Scuseria-Ernzerhof (HSE) XC functional and added Van der Waals interactions. The method is validated using known bandgaps for BLK-P, phosphorene, and other wide-bandgap 2D materials such as hBN. This provides a quantitative understanding of the range of bandgaps of phosphorous allotropes.

The first-principles results provided here indicate clearly that the relative band position between black and violet phosphorous is of Type I, and that the band offset is large enough to create a quantum well only from phosphorous allotropes. In addition, their band position relative to $\mathrm{hBN}$ demonstrates that the latter can be used for efficient electrical insulation with thick layers, or for thin vertical tunneling barriers in 2D phosphorous devices. In addition, our results provide a framework to interpret future characterization data for these material structures, such as that from photoluminescence or vertical electron tunneling experiments.

\section{References}

[1] H. Liu, A. T. Neal, Z. Zhu, Z. Luo, X. Xu, D. Tomnek, and P. D. Ye, "Phosphorene: An unexplored 2d semiconductor with a high hole mobility," ACS Nano, vol. 8, pp. 4033-4041, 2014.

[2] L. Li, Y. Yu, G. J. Ye, Q. Ge, X. Ou, H. Wu, D. Feng, X. H. Chen, and Y. Zhang, "Black phosphorus field-effect transistors," Nat. Nanotechnol., vol. 9, pp. 372 EP, 2014.

[3] J. E. Padilha, A. Fazzio, and A. J. R. da Silva, "van der waals heterostructure of phosphorene and graphene: Tuning the schottky barrier and doping by electrostatic gating," Phys. Rev. Lett., vol. 114, pp. 066803, 2015.

[4] L. Huang, N. Huo, Y. Li, H. Chen, J. Yang, Z. Wei, J. Li, and S.-S. Li, "Electric field tunable band offsets in black phosphorus and mos2 van der waals p-n heterostructure," J. Phys. Chem. Lett., vol. 6, pp. 2483-2488, 2015.

[5] Z. Zhang, D H. Xing, J. Li, Q. Yan, "Hittorf's phosphorus: the missing link during transformation of red phosphorus to black phosphorus," Cryst. Eng. Comm., vol. 6, pp. 905-909, 2017. 